\title{
ON THE POLYNOMIAL SOLUTIONS OF THE GENERALIZED LAME DIFFERENTIAL EQUATION
}

\author{
MORRIS MARDEN
}

1. Introduction. The generalized Lamé differential equation with which we shall be concerned is

$$
P(z) w^{\prime \prime}+Q(z) w^{\prime}+R(z) w=0,
$$

where $P(z), Q(z)$, and $R(z)$ are real polynomials of degrees $p$, at most $p-1$, and at most $p-2$ respectively. We shall assume that $P(z)$ has $p$ distinct zeros $a_{j}=b_{j}+i c_{j}$ of which the first $q, 0<q \leqq p / 2$, lie in the upper half-plane, the next $q$ are respectively the conjugate imaginaries of the first $q$, and the remaining $p-2 q$ (if any) are real. That is,

$$
\begin{array}{ll}
c_{i}>0 & \text { for } 1 \leqq j \leqq q, \\
c_{j}=-c_{j-q} & \text { for } q<j \leqq 2 q,
\end{array}
$$

and, if $2 q<p$,

$$
c_{j}=0 \quad \text { for } 2 q<j \leqq p .
$$

We assume further that $Q(z)$ has no factor in common with $P(z)$ and that in the development

$$
\frac{Q(z)}{P(z)}=\sum_{j=1}^{p} \frac{A_{j} e^{i \alpha_{j}}}{z-a_{j}}
$$

the coefficients satisfy the inequalities

$$
A_{j}>0, \quad\left|\alpha_{i}\right|<\pi / 2 \quad \text { for } 1 \leqq j \leqq p .
$$

According to Heine (see Marden [1, §9], or Marden [2]), ${ }^{1}$ there exist some choices $V(z)$ of the polynomial $R(z)$ corresponding to each of which equation (1.1) has a polynomial solution $S(z)$. The $S(z)$ were called Stieltjes polynomials by Van Vleck in honor of the man who first studied the location of their zeros relative to those of $P(z)$. For a similar reason, we shall call the $V(z)$ Van Vleck polynomials.

In a previous paper (Marden [2]) we obtained the following result:

For each $j=1,2, \cdots, q$ let there be constructed an ellipse $e\left(a_{j}\right)$ with the points $a_{j}$ and $\bar{a}_{j}$ as foci and with $\cos \alpha_{j}$ as eccentricity. Let $K$ be the smallest convex region which encloses both all the ellipses $e\left(a_{j}\right), j$

Presented to the Society, September 7, 1948; received by the editors December 28, 1948 and, in revised form, April 26, 1949.

1 Numbers in brackets refer to the bibliography at the end of the paper. 
$=1,2, \cdots, q$, and all the real points $a_{j}, j=2 q+1,2 q+2, \cdots, p^{2}$ Then all the zeros of every Stieltjes polynomial $S(z)$ and all the zeros of the corresponding Van Vleck polynomial $V(z)$ lie in $K$.

This result is analogous to the theorem of Lucas (see Marden [1, §6]) that all the zeros of the derivative of a polynomial $f(z)$ lie in the smallest convex region containing all the zeros of $f(z)$.

In the present paper on the zeros of the $S(z)$ and $V(z)$, we propose to develop some results which will be analogous to the following theorem of Jensen (see Marden [1, §7]).

Every non-real zero of the derivative of a real polynomial $f(z)$ lies in or on at least one of the circles (called the Jensen circles of $f(z)$ ) which has as diameter the line segment joining a pair of conjugate imaginary zeros of $f(z)$.

We propose to develop these results by a combination of the methods introduced in Walsh [1] and Marden [2 and 3].

2. Two lemmas. Let us denote by $n$ the degree of $S(z)$, by $k$ the number of pairs of conjugate imaginary zeros of $S(z)$, by $z_{j}=x_{j}+i y_{j}$ $(j=1,2, \cdots, k)$ the zeros of $S(z)$ in the upper half-plane, and, if $k<n / 2$, by $z_{j}(j=2 k+1,2 k+2, \cdots, n)$ the real zeros of $S(z)$. Thus,

$$
S(z)=\left(z-z_{1}\right)\left(z-\bar{z}_{1}\right) \cdots\left(z-z_{k}\right)\left(z-\bar{z}_{k}\right)\left(z-z_{2 k+1}\right) \cdots\left(z-z_{n}\right) .
$$

Also, let us associate with each pair $a_{j}, \bar{a}_{j}(j=1,2, \cdots, q)$ an ellipse $E(j, k)$ defined as follows. Its center will be at the point $\boldsymbol{\gamma}_{j}$ on the real axis such that angle $\bar{a}_{j}, a_{j}, \gamma_{j}$ is equal to $\alpha_{j}$; that is,

$$
\gamma_{j}=b_{j}+c_{j} \tan \alpha_{j} \text {. }
$$

Its minor axis will be parallel to the $y$-axis and be of length $m_{j}$ $=2\left|a_{j}-\gamma_{j}\right|$, and its major axis will be of length $\left(k^{1 / 2} m_{j}\right)$.

We shall now state two lemmas concerning these ellipses.

LEMMA 1. The envelope of all the circles whose diameters are the vertical chords of the ellipse $E(j, k)$ is the ellipse $E(j, k+1)$.

As this lemma may be established by elementary calculus, we shall proceed to the next lemma.

Lemma 2. If a non-real zero $z_{1}$ of $S(z)$ lies exterior to all the circles $E(j, 1), j=1,2, \cdots, q$, then it lies inside at least one of the Jensen circles $J\left(z_{u}\right)$ of $S(z), u=2,3, \cdots, k$.

Proof. According to equation $(9,3)$ in Marden [1], the zero $z_{1}$, not being a point $a_{j}$, satisfies the equation

2 If $p \leqq 2 q$, reference to real points $a_{j}$ should be omitted. 


$$
\sum_{j=1}^{p} \frac{\alpha_{j}}{z_{1} a_{j}}+\sum_{j=2}^{n} \frac{2}{z_{1}-z_{j}}=0
$$

that is,

$$
\sum_{j=1}^{q} U_{i}+\sum_{j=2 q+1}^{p} V_{i}+\sum_{j=2}^{k} X_{i}+\sum_{j=2 k+1}^{n} Y_{j}+Z=0
$$

where

$$
\begin{aligned}
U_{i} & =A_{j}\left[e^{i \alpha_{j}}\left(z_{1}-a_{j}\right)^{-1}+e^{-i \alpha_{j}}\left(z_{1}-\bar{a}_{j}\right)^{-1}\right], \\
V_{i} & =A_{i}\left(z_{1}-a_{j}\right)^{-1}, \\
X_{j} & =2\left[\left(z_{1}-z_{j}\right)^{-1}+\left(z_{1}-\bar{z}_{j}\right)^{-1}\right], \\
Y_{j} & =2\left(z_{1}-z_{j}\right)^{-1}, \\
Z & =2\left(z_{1}-\bar{z}_{1}\right)^{-1} .
\end{aligned}
$$

Let us compute the sign of the imaginary part of each term in equation (2.2). From the equation

$$
\begin{aligned}
2 i \operatorname{Im} U_{i}= & A_{j}\left\{e^{i \alpha_{j}}\left[\left(z_{1}-a_{j}\right)^{-1}-\left(\bar{z}_{1}-a_{j}\right)^{-1}\right]\right. \\
& \left.+e^{-i \alpha_{j}}\left[\left(z_{1}-\bar{a}_{j}\right)^{-1}-\left(\bar{z}_{1}-\bar{a}_{j}\right)^{-1}\right]\right\} \\
=-2 i y_{1} A_{j}\left\{e^{i \alpha_{j}}\left[\left(z_{1}-a_{j}\right)\left(\bar{z}_{1}-a_{j}\right)\right]^{-1}\right. & \\
& \left.+e^{-i \alpha_{j}}\left[\left(z_{1}-\bar{a}_{j}\right)\left(\bar{z}_{1}-\bar{a}_{j}\right)\right]^{-1}\right\},
\end{aligned}
$$

it follows that

$\operatorname{sg} \operatorname{Im} U_{j}=-\operatorname{sg}\left(y_{1} \operatorname{Re}\left\{e^{i \alpha_{j}}\left[\left(x_{1}-b_{j}\right)+i\left(y_{1}+c_{j}\right)\right]\left[\left(x_{1}-b_{j}\right)-i\left(y_{1}-c_{j}\right)\right]\right\}\right)$

$$
\begin{aligned}
& =-\operatorname{sg}\left\{y_{1}\left[\left(x_{1}-b_{j}\right)^{2}+y_{1}^{2}-c_{j}^{2}-2 c_{j}\left(x_{1}-b_{j}\right) \tan \alpha_{j}\right] \cos \alpha_{i}\right\} \\
& =-\operatorname{sg}\left\{y_{1}\left[\left(x_{1}-\gamma_{j}\right)^{2}+y_{1}^{2}-c_{i}^{2} \sec ^{2} \alpha_{i}\right]\right\} .
\end{aligned}
$$

From the definitions of $V_{j}$ and $Z$, it follows at once that

$$
\operatorname{sg} \operatorname{Im} V_{j}=-\operatorname{sg} y_{1} \text { and } \operatorname{sg} \operatorname{Im} Z=-\operatorname{sg} y_{1} \text {. }
$$

To obtain $\operatorname{Im} X_{j}$, we need only in $U_{j}$ replace $a_{j}$ by $z_{j}$ and $A_{j} e^{\alpha_{j} i}$ by unity. Similarly, to obtain $\operatorname{Im} Y_{j}$ we need only in $V_{j}$ replace $a_{j}$ by $z_{j}$. Thus,

$\operatorname{sg} \operatorname{Im} X_{j}=-\operatorname{sg}\left\{y_{1}\left[\left(x_{1}-x_{i}\right)^{2}+y_{1}^{2}-y_{i}^{2}\right]\right\}$,

$\operatorname{sg} \operatorname{Im} Y_{i}=-\operatorname{sg} y_{1}$.

If, now, we suppose the zero $z_{1}$ of $S(z)$ to lie outside both all the circles $E(j, 1), j=1,2, \cdots, q$, and all the Jensen circles $J\left(z_{j}\right)$ of 
$S(z), j=2,3, \cdots, k$, then the imaginary part of each term on the left side of equation (2.2) would have the same sign, namely $s g\left(-y_{1}\right)$. That is, the left side of equation (2.2) would not vanish. Hence, if all the $U_{j}$ have the sign $s g\left(-y_{1}\right)$, at least one $X_{j}$ must have the opposite sign. That is, if $z_{1}$ is outside all the circles $E(j, 1)$, it lies inside at least one circle $J\left(z_{j}\right)$.

We remark in passing that Lemma 2 may be modified as follows so as to admit the possibility of both positive and negative $A_{j}$ in equation (1.2).

LEMMA $2^{\prime}$. If a non-real zero $z_{1}$ of $S(z)$ lies simultaneously exterior to all the circles $E(j, 1)$ corresponding to positive $A_{j}$ and interior to all the circles $E(j, 1)$ corresponding to negative $A_{j}$, then it lies inside at least one Jensen circle $J\left(Z_{u}\right)$ of $S(z), u=2,3, \cdots, k$.

However, as Lemma $2^{\prime}$ is not suited to iteration, we shall revert to our original hypothesis on the $A_{j}$ and in the following sections we shall use only Lemma 2.

3. The zeros of the Stieltjes polynomials. By applying Lemmas 1 and 2, we shall now construct a proof of the following theorem, which, when $\alpha_{j}=0$ for all $j$, reduces to a result in Walsh [1].

Theorem I. Let $S(z)$ be any Stieltjes polynomial which possesses $k$ pairs of conjugate imaginary zeros. Then each non-real zero of $S(z)$ lies in or on at least one of the ellipses $E(j, k), j=1,2, \cdots, q$.

Let us suppose that the non-real zero $z_{1}$ of $S(z)$ lies outside all the circles $E(j, 1)$. Then, by Lemma 2 it lies inside at least one Jensen circle of $S(z)$, say $J\left(z_{2}\right)$, the circle which has as diameter the linesegment joining $z_{2}$ and $\bar{z}_{2}$. If also $z_{2}$ lies outside all the circles $E(j, 1)$, then it lies in at least one of the remaining Jensen circles of $S(z)$, say $J\left(z_{3}\right)$. If we continue this process, the number of remaining Jensen circles diminishes. Eventually for some $u, u \leqq k$, though $z_{u-1}$ lies outside all the circles $E(j, 1)$ and hence say in $J\left(z_{u}\right)$, the point $z_{u}$ lies inside at least one circle $E(j, 1)$, say $E(1,1)$.

Now, applying Lemma 1 , we infer that $z_{u-1}$ lies in $E(1,2)$; consequently that $z_{u-2}$ lies in $E(1,3)$, and so on, and finally that $z_{1}$ lies in $E(1, u)$.

Since ellipse $E(1, u)$ for $u \leqq k$ is contained in the ellipse $E(1, k)$, we have completed the proof of Theorem 1 .

Concerning the derivatives of $S(z)$, we may state the following theorem.

Theorem II. Let $S^{(r)}(z)$ denote the rth derivative of the Stieltjes 
polynomial defined in Theorem I. Then every non-real zero of $S^{(r)}(z)$ lies in or on at least one of the ellipses $E(j, k+r), j=1,2, \cdots, q$.

To prove Theorem II, let us denote by $z_{j}^{(r)}$ the zeros of $S^{(r)}(z)$. According to Jensen's Theorem, each non-real zero $z_{1}^{(r)}$ lies in at least one Jensen circle of $S^{(r-1)}(z)$, say $J\left(z_{1}^{(r-1)}\right)$. The zero $z_{1}^{(r-1)}$ lies in at least one Jensen circle of $S^{(r-2)}(z)$, say $J\left(z_{1}^{(r-2)}\right)$, and so on. The zero $z_{1}$ must lie in at least one Jensen circle of $S(z)$, say $J\left(z_{1}\right)$. Since, according to Theorem I, $z_{1}$ lies in an ellipse $E(j, k)$, say $E(1, k)$, we learn again by repeated use of Lemma 1 that $z_{1}^{(r)}$ lies in the ellipse $E(1, k+r)$.

Theorems I and II presuppose our knowledge of the number of pairs of conjugate imaginary zeros possessed by $S(z)$. If, however, we know only the degree $n$ of $S(z)$, we may find the following immediate corollary of these theorems more useful.

Corollary 1. Let $S(z)$ be a Stieltjes polynomial of degree $n$. Then every non-real zero of $S(z)$ lies in or on at least one of the ellipses $E(j, n / 2)$ and every non-real zero of $S^{(r)}(z)$ lies in at least one of the ellipses $E(j,(2 r+n) / 2), j=1,2, \cdots, q$. If $n$ is odd, these ellipses may be replaced by the smaller ellipses $E(j,(n-1) / 2)$ and $E(j,(2 r+n-1) / 2)$ respectively.

4. The zeros of the Van Vleck polynomials. We shall now prove the following theorem.

TheOREM III. Let $V(z)$ be the Van Vleck polynomial which corresponds to the Stieltjes polynomial $S(z)$ of Theorem $I$. Then every nonreal zero $t_{1}$ of $V(z)$ lies in or on at least one ellipse $E(j, k+2), j$ $=1,2, \cdots, q$.

Let us consider the following three cases.

A. Point $t_{1}$ lies in or on some circle $E(j, 1)$.

B. It is one of the zeros $z_{1}^{\prime}, z_{2}^{\prime}, \cdots, z_{n-1}^{\prime}$ of $S^{\prime}(z)$.

C. It is neither a point $z_{j}^{\prime}$, nor a point in or on a circle $E(j, 1)$.

The theorem is obviously true in case $A$. In case $\mathrm{B}$, according to Theorem II, $t_{1}$ lies in some ellipse $E(j, k+1)$ and hence in some ellipse $E(j, k+2)$. In case $\mathrm{C}$, we shall use the fact that $t_{1}$ satisfies equation $(9,5)$ of Marden [1]; namely,

$$
\sum_{j=1}^{p} \frac{A_{j} e^{i \alpha_{j}}}{t_{1}-a_{1}}+\sum_{j=1}^{n-1} \frac{1}{t_{1}-z_{j}^{\prime}}=0 .
$$

The left side of (4.1) is comprised of terms of the same form as the $U_{j}, V_{j}, X_{j}$, and $Y_{j}$ of equation (2.2). As in Lemma 2, we conclude therefore that $t_{1}$ must lie in at least one of the Jensen circles of $S^{\prime}(z)$, 
say $J\left(z_{1}^{\prime}\right)$. By Theorem II, $z_{1}^{\prime}$ must lie in at least one of the ellipses $E(j, k+1)$ and hence by Lemma 1 point $t_{1}$ must lie in at least one of the ellipses $E(j, k+2)$. This completes the proof of Theorem III.

If we now employ the same reasoning as for Theorem II, we may infer the following result concerning the derivatives of $V(z)$.

THEOREM IV. Let $V^{(r)}(z)$ denote the rth derivative of the polynomial $V(z)$ of Theorem III. Then every non-real zero of $V^{(r)}(z)$ lies in or on at least one of the ellipses $E(j, k+2+r), j=1,2, \cdots, q$.

Finally, we may infer at once from Theorems III and IV the following complement to Corollary 1.

CoRollary 2. Let $V(z)$ be the Van Vleck polynomial corresponding to a Stieltjes polynomial of degree $n$. Then every non-real zero of $V(z)$ lies in or on at least one of the ellipses $E(j,(n+4) / 2)$ and every nonreal zero of $V^{(r)}(z)$ lies in or on at least one of the ellipses, $E(j,(2 r+n$ $+4) / 2), j=1,2, \cdots, q$. If $n$ is odd, these ellipses may be replaced by the smaller ellipses $E(j,(n+3) / 2)$ and $E(j,(2 r+n+3) / 2)$ respectively.

\section{Bibliography}

\section{MARDEN}

1. The geometry of the zeros of a polynomial in a complex variable, Mathematical Surveys, no. 3, New York, American Mathematical Society, 1949.

2. On Stieltjes polynomials, Trans. Amer. Math. Soc. vol. 33 (1931) pp. 934-944.

3. On the zeros of certain real rational and meromorphic functions, Duke Math. J. vol. 16 (1949) pp. 91-97.

\section{J. L. WALSH}

1. On the location of the roots of Lamé's polynomials, Tôhoku Math. J. vol. 23 (1924) pp. 312-317.

UNIVERSITY OF Wisconsin, MiLWAUKeE 